\title{
Contain your excitement
}

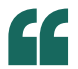

we decided to actively use [coherence] to guide us in changing the structure of the molecule with the aim of altering its photophysics
Efficient conversion of light into chemical potential relies on effective and long-lasting charge separation, whether this occurs in batteries, biosystems or, more generally, in molecules. Inorganic-based chromophores are molecules in which charge separation usually occurs through the transition of one electron from the metal centre to the ligands forming what is known as a metal-to-ligand charge transfer (MLCT) excited state. By exploiting the coupling between vibrational and electronic degrees of freedom involved in this excited state, James McCusker and co-workers report in Nature a design strategy that allows for synthetic control of the lifetime of the MLCT states in iron(II)-based complexes.

Transition-metal chromophores are well known for their photophysical properties and their ability to convert light into chemical potential. For example, the lowestenergy photoexcited state of ruthenium chromophores can store a substantial amount of energy and last up to a microsecond. This would be a lifetime long enough to make these chromophores ideal for technological applications, if only the low availability - and thus high cost - of Ru were not seriously limiting scalability. The obvious solutions would be to use chromophores based on Earth-abundant metals, and iron seems a good alternative. It belongs

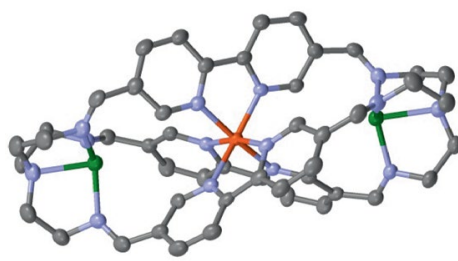

Credit: Adapted from Paulus, B. C. et al. Nature 582, 214-218 (2020), Springer Nature Limited to the same group of the periodic table as Ru, and MLCT excited states of Fe(II)-based chromophores are identical to those of their $\mathrm{Ru}$ analogues, but they have a very short lifetime. "We showed many years ago that, in Fe-based complexes, these states last less than $100 \mathrm{fs}$. This basically undercuts the ability to use Fe-based chromophores in virtually any setting that relies on using the energy stored in a charge-separated state," remarks McCusker.

The group led by McCusker proposed that an understanding of the geometrical distortions associated with the ultrafast decay of the MLCT states in Fe(II)chromophores might enable synthetic alterations resulting in an increased MLCT state lifetime. They specifically looked at an Fe(II) complex using transient absorption spectroscopy to look at some of the very fast (sub-100 fs) vibrational dynamics that occur in such a compound. This technique uses ultrafast pulses that have enough energy to excite multiple vibrations in a molecule, generating a so-called wavepacket. Because all these vibrational states are excited at the same time, they start oscillating at the same time, that is, they are in phase or coherent. The group identified these coherent oscillations for the Fe(II) complex but noticed that they were dephasing too rapidly to be purely vibrational and too slowly to be purely electronic. They therefore hypothesized that these are vibronic coherences that arise from the coupling between vibrational and electronic degrees of freedom. In particular, the observed dephasing times suggested that the vibrational degrees of freedom might be coupled to the decay of the MLCT state. Density-functional theory calculations revealed the vibrational modes at play in this vibronic coupling, which then guided the modification of the compound with the aim of rigidifying that specific vibration and increasing the lifetime of the MLCT state. "In this case, the large amplitude motion in the imine cap of the ligand gave us the idea of inserting a metal ion $(\mathrm{Cu}(\mathrm{I}))$ to lock these motions down," says McCusker. "It worked: the MLCT lifetime increased by a factor of 20."

"The increase in the MLCT lifetime is significant in a relative sense insofar as it is the longest that has been observed for an Fe(II)-polypyridyl system. But, advances using the carbene-based ligand, especially the work of Ken Warnmark at Lund University, far outpaces what we have achieved," explains McCusker. "What is significant in our study is that instead of simply observing the coherence, we decided to actively use this information to guide us in changing the structure of the molecule with the aim of altering its photophysics."

The study shows that it seems possible to synthetically control ultrafast excited-state dynamics, and the approach proposed by McCusker and co-workers should be generally applicable to any chromophore. "It has the potential to eliminate some of the trial-and-error that goes into structure-dynamic correlation studies because it can (potentially) tell you what to focus on right out of the gate," he concludes.

Gabriella Graziano

ORIGINAL ARTICLE Paulus, B. C. et al. Leveraging excited-state coherence for synthetic control of ultrafast dynamics. Nature 582, 214-218 (2020) 